\title{
State-space modeling of modular multilevel converters including line frequency trans- former
}

A. Christe and D. Dujic

This material is posted here with permission of the IEEE. Such permission of the IEEE does not in any way imply IEEE endorsement of any of EPFL's products or services. Internal or personal use of this material is permitted. However, permission to reprint / republish this material for advertising or promotional purposes or for creating new collective works for resale or redistribution must be obtained from the IEEE by writing to pubs-permissions@ieee. org. By choosing to view this document, you agree to all provisions of the copyright laws protecting it. 


\title{
State-Space Modeling of Modular Multilevel Converters Including Line Frequency Transformer
}

\author{
Alexandre Christe, Dražen Dujić \\ Power Electronics Laboratory \\ École Polytechnique Fédérale de Lausanne - EPFL \\ Station 11, CH-1015 Lausanne \\ Email: alexandre.christe@epfl.ch,drazen.dujic@epfl.ch \\ URL: http://pel.epfl.ch
}

\section{Acknowledgments}

This work is part of the Swiss Competence Centers for Energy Research (SCCER) initiative which is supported by the Swiss Commission for Technology and Innovation (CTI).

\section{Keywords}

$<<$ Multilevel Converters $>>,<<$ Transformer $>>,<<$ Modelling $>>$.

\begin{abstract}
This paper presents a detailed state-space modeling of a Modular Multilevel Converter (MMC) in combination with line frequency transformer (LFT). The classical MMC topology is compared with the recently proposed Open-End Winding MMC (OEWMMC) topology, which integrates the LFT in the converter arm. The line frequency transformer parameters are added in the model, some of which are often neglected in the available literature. Both models are verified and compared by means of numerical simulations in open-loop. Despite integration attractiveness offered by the OEWMMC topology, there are also some inherent drawbacks affecting the overall system sizing that are discussed in the paper.
\end{abstract}

\section{Nomenclature}

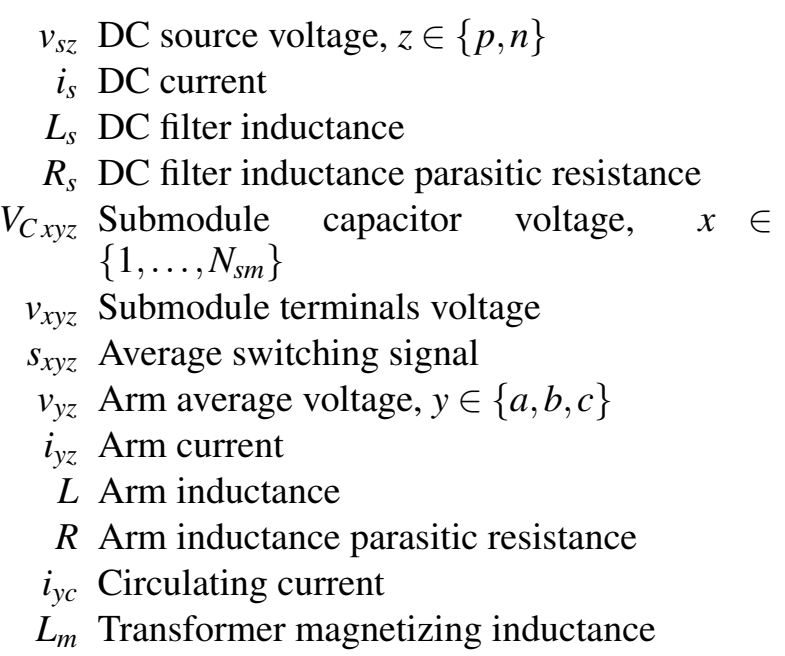

$i_{x m}$ Magnetizing current

$L_{\sigma}$ Transformer leakage inductance

$R_{\sigma}$ Transformer leakage inductance parasitic resistance

$L_{o}$ Grid inductance

$R_{o}$ Grid resistance

$i_{y o}$ Grid phase current

$v_{y}$ Grid phase voltage

$P$ DC positive terminal

$N$ DC negative terminal

$M$ Grid star point

$n$ DC side transformer star connection

$n^{\prime}$ Grid side transformer star connection 


\section{Introduction}

Modular Multilevel Converter (MMC) [1] is under scrutiny of various research groups around the world, and is one of the most active research topic in power electronics over the last decade. This is especially true for HVDC applications, where the MMC has been adopted and commercialized rather quickly by the industry. The main advantages of the MMC are the absence of series-connected devices (or at most only a few) compared to classical Voltage Source Converters (VSCs), the almost unlimited voltage scalability by means of series-connected submodules (SMs), the opportunity to standardize SM and use in a variety of applications, the reduction or cancellation of filtering needs due to increased quality of AC voltage waveform, etc. All this is possible at the expense of increased control effort, communication complexity and an overall converter volume increase, compared to the equivalent state-of-the-art counterparts. Nevertheless, in high power applications, inherent voltage scalability and design modularity are resulting in reevaluation of the competitiveness of some well established technologies, such as Line Commutated Inverters (LCIs) and VSCs. In addition to HVDC, other applications where MMCs are being considered or envisioned, are FACTS [2], grid interties [3], STATCOMs [4], MV drives [5], DC electric ships [6], etc.

Motivation for the work presented in this paper comes from an increased interest in emerging concepts of Medium Voltage DC (MVDC) distribution grids [7], and lack of suitable conversion technology (preferably with galvanic isolation). While concepts such as power electronics transformers [8] or solid state transformers [9] are already being proposed, their multi-stage conversion and increased complexity have to be carefully assessed for each particular application [10]. When targeting a single-stage conversion from DC to AC or vice versa in the MV range, MMCs can be considered as an attractive solution.

The modeling aspects of the MMC have been addressed in many scientific papers. Starting from average models $[11,12]$ where only the average capacitor arm voltage is considered, modeling of MMCs has further evolved to models accounting for the modulation technique [13], including switching events of one cell per arm [14], models in the frequency domain [15], using the phasor theory [16] or being very specific and oriented towards the targeted application (grid connection [17], motor control [18]).

With regard to the transformer integration into the MMC structure, some proposals have been made already, such as those presented in [19, 20], or covered in patent applications [21, 22]. In [19], authors have considered a transformer structure derived from the idea of center-tap arm inductors, and proposed that the transformer should replace the branch inductors at the MMC output. On the other hand, in the work of [20] inspiration has been taken from open-end winding configurations used for motor drives, and transformer winding is directly integrated into the MMC phase leg, between two virtual arms. In this paper we refer to this topology as the Open-End Winding MMC (OEWMMC).

The modeling of the classical MMC presented in this paper includes the parameters of the Low Frequency Transformer (LFT) (i.e. magnetizing branch) through a state-space analysis similar to that in [23]. In the first step, the decoupled current model is derived for the decoupled current control of the MMC. In the second step, the state-space model of the OEWMMC is established and compared with the classical MMC's model. The inherent DC bias in the integrated transformer primary winding is discussed and simulation results are provided for both topologies, in order to highlight similarities and differences.

\section{MMC topologies of interest}

The reference topology for the analysis is the classical MMC connected to an external LFT, as shown in Fig. 1 with all the relevant parameters. The model of a single-phase transformer includes: a magnetizing inductance, an ideal transformer of ratio $N_{1} / N_{2}$, a total equivalent leakage inductance from primary and secondary sides $L_{\sigma}$ as well as a total winding resistance $R_{\sigma}$, both referenced to the secondary side. Such a model is valid (i.e. behaves like a T model) if $L_{m} \gg L_{\sigma 1}, L_{\sigma 2}$. On the other hand, the OEWMMC (Fig. 2) proposed by [20] offers two interesting features: i) the transformer winding is integrated directly in the MMC phase legs and ii) the arm inductors are substituted by the transformer leakage inductance. In addition, the $\mathrm{AC}$ waveform magnitude across the integrated winding is doubled compared to the classical MMC. In other words, only half of the input MVDC voltage, and therefore half the number of cells, is needed to produce the same primary AC voltage magnitude across the transformer winding (taking the LFT from the classical MMC as a reference case). Clearly, these differences directly affect the current ratings of the semiconductors, as shown in Table I. For comparison purposes the, MVDC input voltage is kept identical (1 [p.u.]). 


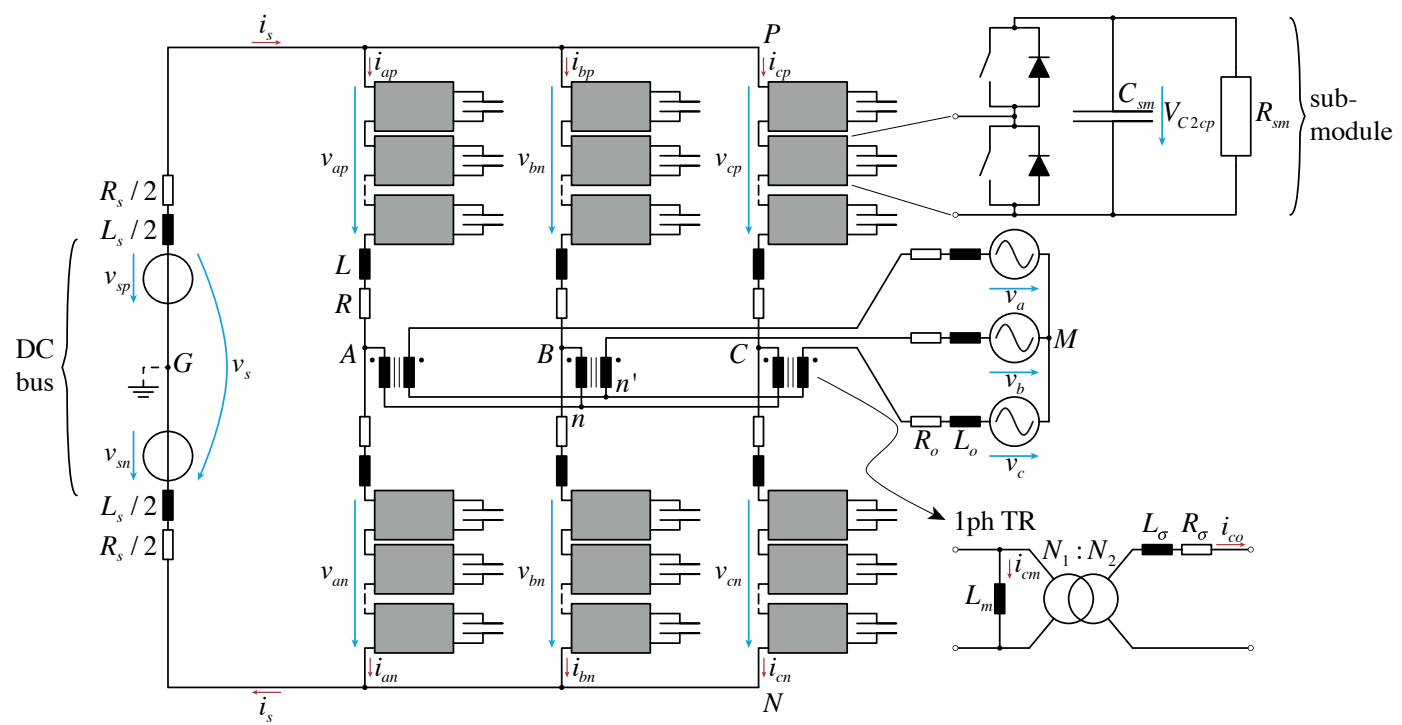

Figure 1: Classical MMC connected to a three-phase transformer. Each arm is composed by $N_{s m}$ series-connected unipolar SMs; in the bottom right part the simplified three single-phase transformer equivalent circuit is shown.

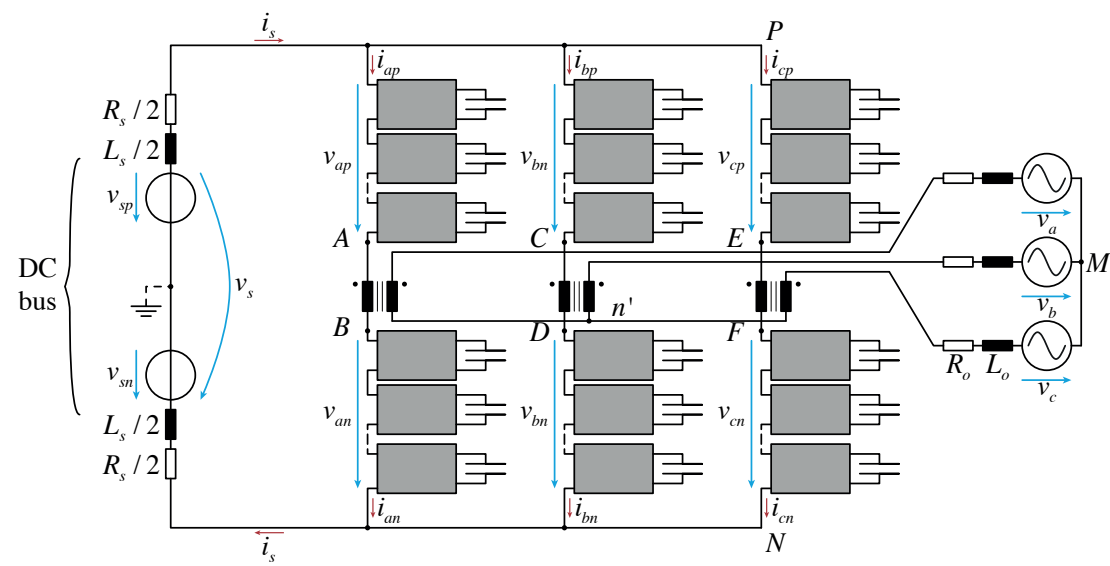

Figure 2: Open-end winding MMC with integrated transformer. Each virtual arm is again composed by $N_{s m}$ series-connected unipolar SMs. For the modeling the same transformer equivalent circuit is used.

Table I: Converter ratings at constant power and unity modulation index.

\begin{tabular}{|l|c|c|c|c|}
\hline & $\begin{array}{c}\text { DC voltage } \\
\text { [p.u.] }\end{array}$ & $\begin{array}{c}\text { DC current } \\
\text { [p.u.] }\end{array}$ & $\begin{array}{c}\text { AC phase voltage } \\
\text { peak [p.u.] }\end{array}$ & $\begin{array}{c}\text { AC phase current } \\
\text { peak [p.u.] }\end{array}$ \\
\hline \hline MMC & 1 & 1 & $1 / 2$ & 1 \\
OEWMMC & 1 & 1 & 1 & $1 / 2$ \\
\hline
\end{tabular}

\section{State-space modeling}

To develop a state-space model, one needs to isolate the derivative terms related to the state variables of the system into the form: $\dot{x}=A x+B u$ ( $+D w$, if there is a disturbance). While the state-space modeling can either deal with scalar or vector variables, for simplicity of presentation, the different variables of the system are defined in vector form ( $\mathbf{v}_{s}$ and $\mathbf{i}_{s}$ are arbitrarily expanded to three components vectors):

$$
\mathbf{v}_{s}=\left[\begin{array}{l}
v_{s} \\
v_{s} \\
v_{s}
\end{array}\right] \mathbf{i}_{s}=\left[\begin{array}{l}
i_{s} \\
i_{s} \\
i_{s}
\end{array}\right] \mathbf{v}_{p, n}=\left[\begin{array}{l}
v_{a p, n} \\
v_{b p, n} \\
v_{c p, n}
\end{array}\right] \mathbf{i}_{p, n}=\left[\begin{array}{l}
i_{a p, n} \\
i_{b p, n} \\
i_{c p, n}
\end{array}\right] \mathbf{v}_{g}=\left[\begin{array}{c}
v_{a} \\
v_{b} \\
v_{c}
\end{array}\right] \mathbf{i}_{o}=\left[\begin{array}{l}
i_{a o} \\
i_{b o} \\
i_{c o}
\end{array}\right] \mathbf{i}_{m}=\left[\begin{array}{l}
i_{a m} \\
i_{b m} \\
i_{c m}
\end{array}\right]
$$


where $\mathbf{v}_{s}$ is the DC source voltage, $\mathbf{i}_{s}$ is the input current on the DC side, $\mathbf{v}_{p}$ and $\mathbf{v}_{n}$ are the arm voltages, $\mathbf{i}_{p}$ and $\mathbf{i}_{n}$ are the arm currents, $\mathbf{v}_{g}$ is the AC grid voltage and $\mathbf{i}_{o}$ is the output AC current.

Two useful matrices that help describing the systems are introduced as in [23]:

$$
\mathbb{P}=\frac{1}{3}\left[\begin{array}{lll}
1 & 1 & 1 \\
1 & 1 & 1 \\
1 & 1 & 1
\end{array}\right] \quad \mathbb{Q}=\frac{1}{3}\left[\begin{array}{ccc}
2 & -1 & -1 \\
-1 & 2 & -1 \\
-1 & -1 & 2
\end{array}\right]
$$

These matrices come along with properties useful for simplification, considering that $\mathbb{P}+\mathbb{Q}=\mathbb{I}$ and $\mathbb{P} \mathbb{Q}=\mathbf{0}$, where $\mathbb{I}$ is the identity matrix and $\mathbf{0}$ is the null matrix. Moreover, additional simplifications apply as well: $\mathbb{Q} \mathbf{i}_{s}=0, \mathbb{Q} \mathbf{v}_{s}=0, \mathbb{P} \mathbf{i}_{o}=0$ and $\mathbb{P} \mathbf{v}_{g}=0$. This means the DC component can be extracted from any variable by a multiplication with $\mathbb{P}$. Alternatively, the AC component of any variable is obtained through a multiplication with $\mathbb{Q}$. For the modeling, it is assumed that an arm balancing control is in place. As the SM voltage dynamics are governed by the same differential equations for both classical MMC and OEWMMC (SM type dependent), the relevant equations are presented only once. The continuous time switching function $s(t)$ applied to one SM (unipolar type) has a range from 0 to 1 . Each SM terminal voltage $\left(v_{x y z}\right)$ is defined next, along with the sum of all SM terminal voltages as:

$$
v_{x y z}(t)=s_{x y z}(t) V_{C x y z}(t) \Rightarrow v_{y z}(t)=\sum_{x=1}^{N} s_{x y z}(t) V_{C x y z}(t)=\mathbf{s}_{y z}^{T} \mathbf{v}_{d c}
$$

Please note that $\mathbf{v}_{d c}$ is the vector containing all SM capacitor voltages, thus $\mathbf{s}_{y z}^{T}$ has to be able to capture only the average voltage of one arm, while $\mathbf{v}_{d c}^{\Sigma}$ is defined as the summed capacitor voltages per arm.

\section{State-space modeling of the classical MMC}

The presented modeling is slightly different from that of [23], as the presence of the LFT is taken into account. The state variables of the system are all SM capacitor voltages, two arm currents and the magnetizing current (small impact on the dynamics). With input current $i_{s}$ split equally between each phase leg (i.e. $\mathbf{i}_{s}=3 \mathbb{P} \mathbf{i}_{p}=3 \mathbb{P} \mathbf{i}_{n}$ ), equations for the input, output and magnetizing current loops are:

$$
\begin{aligned}
& \left\{\begin{array}{l}
\mathbf{v}_{s}=\left(3 L_{s} \mathbb{P}+L\right) \frac{\mathrm{d}}{\mathrm{d} t} \mathbf{i}_{p}+\left(3 R_{s} \mathbb{P}+R\right) \mathbf{i}_{p}+\mathbf{v}_{p}+L \frac{\mathrm{d}}{\mathrm{d} t} \mathbf{i}_{n}+R \mathbf{i}_{n}+\mathbf{v}_{n} \\
\mathbf{v}_{s}=\left(3 L_{s} \mathbb{P}+L\right) \frac{\mathrm{d}}{\mathrm{d} t} \mathbf{i}_{n}+\left(3 R_{s} \mathbb{P}+R\right) \mathbf{i}_{n}+\mathbf{v}_{p}+L \frac{\mathrm{d}}{\mathrm{d} t} \mathbf{i}_{p}+R \mathbf{i}_{p}+\mathbf{v}_{n}
\end{array}\right. \\
& \left\{\begin{array}{l}
\mathbb{Q} \mathbf{v}_{p}+L \mathbb{Q} \frac{\mathrm{d}}{\mathrm{d} t} \mathbf{i}_{p}+R \mathbb{Q} \mathbf{i}_{p}+L_{m} \frac{\mathrm{d}}{\mathrm{d} t} \mathbf{i}_{m}=0 \\
\mathbb{Q} \mathbf{v}_{n}+L \mathbb{Q} \frac{\mathrm{d}}{\mathrm{d} t} \mathbf{i}_{n}+R \mathbb{Q} \mathbf{i}_{n}-L_{m} \frac{\mathrm{d}}{\mathrm{d} t} \mathbf{i}_{m}=0
\end{array}\right. \\
& L_{m} \frac{\mathrm{d}}{\mathrm{d} t} \mathbf{i}_{m}=\left(L_{o}^{\prime}+L_{\sigma}^{\prime}\right)\left(\frac{\mathrm{d}}{\mathrm{d} t} \mathbf{i}_{p}-\frac{\mathrm{d}}{\mathrm{d} t} \mathbf{i}_{n}-\frac{\mathrm{d}}{\mathrm{d} t} \mathbf{i}_{m}\right)+\left(R_{o}^{\prime}+R_{\sigma}^{\prime}\right)\left(\mathbf{i}_{p}-\mathbf{i}_{n}-\mathbf{i}_{m}\right)+\mathbf{v}_{g}^{\prime}
\end{aligned}
$$

Starting from the reference set of equations, the independent current derivatives are obtained as:

$$
\begin{aligned}
\frac{\mathrm{d}}{\mathrm{d} t} \mathbf{i}_{p}= & \frac{1}{2 L+3 L_{s}}\left(\mathbf{v}_{s}-\mathbb{P}\left(\mathbf{v}_{p}+\mathbf{v}_{n}\right)-\left(3 R_{s}+2 R\right) \mathbb{P} \mathbf{i}_{p}\right)-\frac{L_{m}+L_{o}^{\prime}+L_{\sigma}^{\prime}}{L L_{m}+\left(L_{o}^{\prime}+L_{\sigma}^{\prime}\right)\left(L+2 L_{m}\right)}\left(R \mathbb{Q} \mathbf{i}_{p}\right. \\
& \left.+\mathbb{Q} \mathbf{v}_{p}\right)-\frac{L_{m}\left(L_{o}^{\prime}+L_{\sigma}^{\prime}\right)}{L\left(L L_{m}+\left(L_{o}^{\prime}+L_{\sigma}^{\prime}\right)\left(L+2 L_{m}\right)\right)}\left(\mathbb{Q}\left(\mathbf{v}_{p}+\mathbf{v}_{n}\right)+R \mathbb{Q}\left(\mathbf{i}_{p}+\mathbf{i}_{n}\right)\right) \\
& -\frac{L_{m}}{L L_{m}+\left(L_{o}^{\prime}+L_{\sigma}^{\prime}\right)\left(L+2 L_{m}\right)}\left(\left(R_{o}^{\prime}+R_{\sigma}^{\prime}\right)\left(\mathbf{i}_{p}-\mathbf{i}_{n}-\mathbf{i}_{m}\right)+\mathbf{v}_{o}^{\prime}\right) \\
\frac{\mathrm{d}}{\mathrm{d} t} \mathbf{i}_{n}= & \frac{1}{2 L+3 L_{s}}\left(\mathbf{v}_{s}-\mathbb{P}\left(\mathbf{v}_{p}+\mathbf{v}_{n}\right)-\left(3 R_{s}+2 R\right) \mathbb{P} \mathbf{i}_{p}\right)-\frac{L_{m}+L_{o}^{\prime}+L_{\sigma}^{\prime}}{L L_{m}+\left(L_{o}^{\prime}+L_{\sigma}^{\prime}\right)\left(L+2 L_{m}\right)}\left(R \mathbb{Q} \mathbf{i}_{n}\right. \\
& \left.+\mathbb{Q} \mathbf{v}_{n}\right)-\frac{L_{m}\left(L_{o}^{\prime}+L_{\sigma}^{\prime}\right)}{L\left(L L_{m}+\left(L_{o}^{\prime}+L_{\sigma}^{\prime}\right)\left(L+2 L_{m}\right)\right)}\left(\mathbb{Q}\left(\mathbf{v}_{p}+\mathbf{v}_{n}\right)+R \mathbb{Q}\left(\mathbf{i}_{p}+\mathbf{i}_{n}\right)\right) \\
& +\frac{L_{m}}{L L_{m}+\left(L_{o}^{\prime}+L_{\sigma}^{\prime}\right)\left(L+2 L_{m}\right)}\left(\left(R_{o}^{\prime}+R_{\sigma}^{\prime}\right)\left(\mathbf{i}_{p}-\mathbf{i}_{n}-\mathbf{i}_{m}\right)+\mathbf{v}_{g}^{\prime}\right)
\end{aligned}
$$




$$
\begin{aligned}
\frac{\mathrm{d}}{\mathrm{d} t} \mathbf{i}_{m}= & -\left(\frac{L_{o}^{\prime}+L_{\sigma}^{\prime}}{L L_{m}+\left(L_{o}^{\prime}+L_{\sigma}^{\prime}\right)\left(L+2 L_{m}\right)}\left(R \mathbb{Q}\left(\mathbf{i}_{p}-\mathbf{i}_{n}\right)+\mathbb{Q}\left(\mathbf{v}_{p}-\mathbf{v}_{n}\right)\right)\right. \\
& \left.-\frac{L}{L L_{m}+\left(L_{o}^{\prime}+L_{\sigma}^{\prime}\right)\left(L+2 L_{m}\right)}\left(\mathbf{v}_{g}^{\prime}+\left(R_{o}^{\prime}+R_{\sigma}^{\prime}\right)\left(\mathbf{i}_{p}-\mathbf{i}_{n}-\mathbf{i}_{m}\right)\right)\right)
\end{aligned}
$$

To get the decoupled current model, different properties of the components are analyzed. Since the selected model is more complex than the one presented in [23], the number of current components is not the same. The arm currents are decomposed as:

$$
\left\{\begin{array}{l}
\mathbf{i}_{p}=\frac{1}{3} \mathbf{i}_{s}+\frac{1}{2} \mathbf{i}_{c}+\frac{1}{2} \tilde{\mathbf{i}}_{o} \\
\mathbf{i}_{n}=\frac{1}{3} \mathbf{i}_{s}+\frac{1}{2} \mathbf{i}_{c}-\frac{1}{2} \tilde{\mathbf{i}}_{o}
\end{array}\right.
$$

where $\tilde{\mathbf{i}}_{o}=\mathbf{i}_{p}-\mathbf{i}_{n}$. The input current which is equally split between the three phase legs, i.e. the DC current flowing through the arms, is given by:

$$
\begin{aligned}
\mathbf{i}_{s} / 3 & =\mathbb{P} \mathbf{i}_{p}=\mathbb{P} \mathbf{i}_{n} \\
\frac{\mathrm{d}}{\mathrm{d} t} \mathbf{i}_{s} / 3 & =\frac{1}{2 L+3 L_{s}}(\mathbf{v}_{s}-\mathbb{P}\left(\mathbf{v}_{p}+\mathbf{v}_{n}\right)-\left(3 R_{s}+2 R\right) \underbrace{\mathbb{P} \mathbf{i}_{p}}_{\mathbf{i}_{s} / 3})
\end{aligned}
$$

The circulating current, i.e. the common $\mathrm{AC}$ current (multiple frequencies) going through each arm of the same phase leg is described as:

$$
\begin{aligned}
\mathbf{i}_{c} & =\mathbb{Q}\left(\mathbf{i}_{p}+\mathbf{i}_{n}\right) \\
\frac{\mathrm{d}}{\mathrm{d} t} \mathbf{i}_{c} & =-\frac{1}{L}(\mathbb{Q}\left(\mathbf{v}_{p}+\mathbf{v}_{n}\right)+R \underbrace{\mathbb{Q}\left(\mathbf{i}_{p}+\mathbf{i}_{n}\right)}_{\mathbf{i}_{c}})
\end{aligned}
$$

Finally, the output current component, i.e. the AC grid current, is:

$$
\begin{aligned}
\mathbf{i}_{o}^{\prime}= & \mathbf{i}_{p}-\mathbf{i}_{n}-\mathbf{i}_{m}=\tilde{\mathbf{i}}_{o}-\mathbf{i}_{m} \\
\frac{\mathrm{d}}{\mathrm{d} t} \mathbf{i}_{o}^{\prime}= & -\frac{L_{m}}{L L_{m}+\left(L_{o}^{\prime}+L_{\sigma}^{\prime}\right)\left(L+2 L_{m}\right)}\left(R \mathbb{Q}\left(\mathbf{i}_{p}-\mathbf{i}_{n}\right)+\mathbb{Q}\left(\mathbf{v}_{p}-\mathbf{v}_{n}\right)\right) \\
& -\frac{2 L_{m}+L}{L L_{m}+\left(L_{o}^{\prime}+L_{\sigma}^{\prime}\right)\left(L+2 L_{m}\right)}\left(\mathbf{v}_{g}^{\prime}+\left(R_{o}^{\prime}+R_{\sigma}^{\prime}\right)(\underbrace{\left(\mathbf{i}_{p}-\mathbf{i}_{n}-\mathbf{i}_{m}\right.}_{\mathbf{i}_{o}^{\prime}})\right)
\end{aligned}
$$

These considerations are relevant for understanding the behavior of the MMC connected to a transformer. Any current taken from the phase leg mid-point, either AC or DC, will come equally from both positive and negative arms (as long as the circulating current is kept close to zero). In the studied configuration, as the grid star point is not connected to the DC bus mid-point, a common mode current can't establish. The dynamics of $\mathbf{i}_{o}^{\prime}$ and $\mathbf{i}_{m}$, considering two extreme cases: i) infinite magnetizing inductance and ii) infinite grid impedance, result into:

1. Infinite magnetizing inductance condition, i.e. $\lim _{L_{m} \rightarrow \infty}$ :

$$
\begin{aligned}
\frac{\mathrm{d}}{\mathrm{d} t} \mathbf{i}_{p}= & \frac{1}{2 L+3 L_{s}}\left(\mathbf{v}_{s}-\mathbb{P}\left(\mathbf{v}_{p}+\mathbf{v}_{n}\right)-\left(3 R_{s}+2 R\right) \mathbb{P} \mathbf{i}_{p}\right)-\frac{1}{L+2\left(L_{o}^{\prime}+L_{\sigma}^{\prime}\right)}\left(R \mathbb{Q} \mathbf{i}_{p}+\mathbb{Q} \mathbf{v}_{p}\right) \\
& -\frac{L_{o}^{\prime}+L_{\sigma}^{\prime}}{L\left(L+2\left(L_{o}^{\prime}+L_{\sigma}^{\prime}\right)\right)}\left(\mathbb{Q}\left(\mathbf{v}_{p}+\mathbf{v}_{n}\right)+R \mathbb{Q}\left(\mathbf{i}_{p}+\mathbf{i}_{n}\right)\right) \\
& -\frac{1}{L+2\left(L_{o}^{\prime}+L_{\sigma}^{\prime}\right)}\left(\mathbf{v}_{g}^{\prime}+\left(R_{o}^{\prime}+R_{\sigma}^{\prime}\right)\left(\mathbf{i}_{p}-\mathbf{i}_{n}\right)\right) \\
\frac{\mathrm{d}}{\mathrm{d} t} \mathbf{i}_{m}= & 0
\end{aligned}
$$




$$
\frac{\mathrm{d}}{\mathrm{d} t} \mathbf{i}_{o}^{\prime}=\frac{-1}{L+2\left(L_{o}^{\prime}+L_{\sigma}^{\prime}\right)}\left(\left(R+2\left(R_{o}^{\prime}+R_{\sigma}^{\prime}\right)\right) \mathbb{Q}\left(\mathbf{i}_{p}-\mathbf{i}_{n}\right)+\mathbb{Q}\left(\mathbf{v}_{p}-\mathbf{v}_{n}\right)+2 \mathbf{v}_{g}^{\prime}\right)
$$

2. Infinite grid impedance condition, i.e. $\lim _{L_{o}^{\prime} \rightarrow \infty}$ and $\lim _{R_{o}^{\prime} \rightarrow \infty}$ :

$$
\begin{aligned}
\frac{\mathrm{d}}{\mathrm{d} t} \mathbf{i}_{p}= & \frac{1}{2 L+3 L_{s}}\left(\mathbf{v}_{s}-\mathbb{P}\left(\mathbf{v}_{p}+\mathbf{v}_{n}\right)-\left(3 R_{s}+2 R\right) \mathbb{P} \mathbf{i}_{p}\right)-\frac{1}{L+2 L_{m}}\left(R \mathbb{Q} \mathbf{i}_{p}+\mathbb{Q} \mathbf{v}_{p}\right) \\
& -\frac{L_{m}}{L\left(L+2 L_{m}\right)}\left(\mathbb{Q}\left(\mathbf{v}_{p}+\mathbf{v}_{n}\right)+R \mathbb{Q}\left(\mathbf{i}_{p}+\mathbf{i}_{n}\right)\right)-\frac{L_{m}}{L+2 L_{m}}\left(\mathbf{i}_{p}-\mathbf{i}_{n}-\mathbf{i}_{m}\right) \\
\frac{\mathrm{d}}{\mathrm{d} t} \mathbf{i}_{m}= & \frac{-1}{L+2 L_{m}}\left(R \mathbb{Q}\left(\mathbf{i}_{p}-\mathbf{i}_{n}\right)+\mathbb{Q}\left(\mathbf{v}_{p}-\mathbf{v}_{n}\right)\right) \\
\frac{\mathrm{d}}{\mathrm{d} t} \mathbf{i}_{o}^{\prime}= & 0
\end{aligned}
$$

In the first case, the expression for $\frac{\mathrm{d}}{\mathrm{d} t} \mathbf{i}_{p}$ is the same as reported in [23]. From a phase leg point of view, the magnetizing current is seen as a purely inductive load current. From the control point of view, the dynamics of the magnetizing current will not be considered, as its amplitude remains almost constant as long as the grid voltage deviation is small, which is normally true. Nevertheless, detailed model is found to be useful for the overall system design, which is beyond the scope of the paper.

\section{State-space modeling of OEWMMC}

Identical to the previous case, the state variables are all the SM capacitor voltages, the arm current and the magnetizing current. The set of reference equations for the currents is given as:

$$
\begin{aligned}
& \mathbf{v}_{s}=3 L_{s} \mathbb{P} \frac{\mathrm{d}}{\mathrm{d} t} \mathbf{i}_{p}+3 R_{s} \mathbb{P} \mathbf{i}_{p}+\mathbf{v}_{p}+L_{m} \frac{\mathrm{d}}{\mathrm{d} t} \mathbf{i}_{m}+\mathbf{v}_{n} \\
& \left(L_{\sigma}^{\prime}+L_{o}^{\prime}+L_{m} \mathbb{Q}\right) \frac{\mathrm{d}}{\mathrm{d} t} \mathbf{i}_{m}=\left(L_{\sigma}^{\prime}+L_{o}^{\prime}\right) \frac{\mathrm{d}}{\mathrm{d} t} \mathbf{i}_{p}+\left(R_{\sigma}^{\prime}+R_{o}^{\prime}\right)\left(\mathbf{i}_{p}-\mathbf{i}_{m}\right)+\mathbf{v}_{g}^{\prime}
\end{aligned}
$$

After some manipulations, the independent current derivatives are obtained:

$$
\begin{array}{r}
\frac{\mathrm{d}}{\mathrm{d} t} \mathbf{i}_{p}=\frac{1}{L_{m}+3 L_{s}}\left(\mathbf{v}_{s}-\mathbb{P}\left(\mathbf{v}_{p}+\mathbf{v}_{n}\right)-3 R_{s} \mathbb{P} \mathbf{i}_{p}\right)-\frac{1}{L_{o}^{\prime}+L_{\sigma}^{\prime}}\left(\frac{L_{m}+L_{o}^{\prime}+L_{\sigma}^{\prime}}{L_{m}} \mathbb{Q}\left(\mathbf{v}_{p}+\mathbf{v}_{n}\right)\right. \\
\left.+\left(R_{\sigma}^{\prime}+R_{o}^{\prime}\right) \mathbb{Q}\left(\mathbf{i}_{p}-\mathbf{i}_{m}\right)+\mathbf{v}_{g}^{\prime}\right) \\
\frac{\mathrm{d}}{\mathrm{d} t} \mathbf{i}_{m}=-\frac{1}{L_{m}} \mathbb{Q}\left(\mathbf{v}_{p}+\mathbf{v}_{n}\right)+\frac{1}{L_{m}+3 L_{s}}\left(\mathbf{v}_{s}-\mathbb{P}\left(\mathbf{v}_{p}+\mathbf{v}_{n}\right)-3 R_{s} \mathbb{P} \mathbf{i}_{p}\right)
\end{array}
$$

The current decomposition is different in the OEWMMC case, considering that there is no phase leg mid-point output between the arms:

$$
\mathbf{i}_{p}=\frac{1}{3} \mathbf{i}_{s}+\mathbf{i}_{c}+\mathbf{i}_{o}^{\prime} \quad \mathbf{i}_{m}=\frac{1}{3} \mathbf{i}_{s}+\mathbf{i}_{c}
$$

Finally, the three current components of the decoupled current model (same as in the classical MMC) are:

$$
\begin{aligned}
\frac{\mathrm{d}}{\mathrm{d} t} \mathbf{i}_{s} / 3 & =\frac{1}{L_{m}+3 L_{s}}\left(\mathbf{v}_{s}-\mathbb{P}\left(\mathbf{v}_{p}+\mathbf{v}_{n}\right)-3 R_{s} \mathbb{P} \mathbf{i}_{p}\right) \\
\frac{\mathrm{d}}{\mathrm{d} t} \mathbf{i}_{c} & =-\frac{1}{L_{m}} \mathbb{Q}\left(\mathbf{v}_{p}+\mathbf{v}_{n}\right) \\
\frac{\mathrm{d}}{\mathrm{d} t} \mathbf{i}_{o}^{\prime} & =-\frac{1}{L_{o}^{\prime}+L_{\sigma}^{\prime}} \mathbb{Q}\left(\mathbf{v}_{p}+\mathbf{v}_{n}\right)-\frac{R_{o}^{\prime}+R_{\sigma}^{\prime}}{L_{o}^{\prime}+L_{\sigma}^{\prime}} \mathbb{Q}(\underbrace{\mathbf{i}_{p}-\mathbf{i}_{m}}_{\mathbf{i}_{o}^{\prime}})-\frac{1}{L_{o}^{\prime}+L_{\sigma}^{\prime}} \mathbf{v}_{g}^{\prime}
\end{aligned}
$$

with $\mathbf{i}_{s} / 3=\mathbb{P} \mathbf{i}_{p}, \mathbf{i}_{c}=\mathbf{i}_{m}-\mathbf{i}_{s} / 3$ and $\mathbf{i}_{o}^{\prime}=\mathbf{i}_{p}-\mathbf{i}_{m}$. 


\section{Remarks regarding the derived models}

A common system level state-space representation illustrates the cascaded connection of the two statespace models in Fig. 3. The different state-space matrices are built from Eqs. (7) to (9), (25) and (26), and there is no direct feed through of the input.

The current distribution is illustrated in Fig. 4. As a consequence of the LFT integration in the OEWMMC, the reference waveforms are different than for the classical MMC (cf. Fig. 5). The most important difference is the current seen by the magnetizing inductance, which will strongly influence the transformer design. In the classical case, since the transformer is in a star connection on the primary side, no DC current will flow through. In the OEWMMC case, both circulating current and one third of the source DC current flow through the magnetizing inductance. The circulating current component is not critical, as it contains harmonics greater or equal than the line frequency. Depending on the control strategy, the capacitor voltage ripple of each SM can be reduced. In one scenario, a circulating current suppression controller [24] could be used to suppress the second harmonic component coming from the paralleling of voltage sources of different amplitudes. Alternatively, injection of additional harmonic components with specific phase and amplitude $[25,26]$ can be used to further reduce the SM capacitor voltage ripple, compensating for the inherent single phase behavior of the converter. However, the situation is different regarding the DC source current, since its value depends on the loading condition $\left(P_{D C}=v_{s} i_{s} \simeq P_{A C}\right)$. In the OEWMMC case, the transformer design would have therefore to handle this DC bias current, resulting in poor utilization of the magnetic material (limited peak to peak flux density) and significant oversizing of the transformer compared to the classical MMC case.

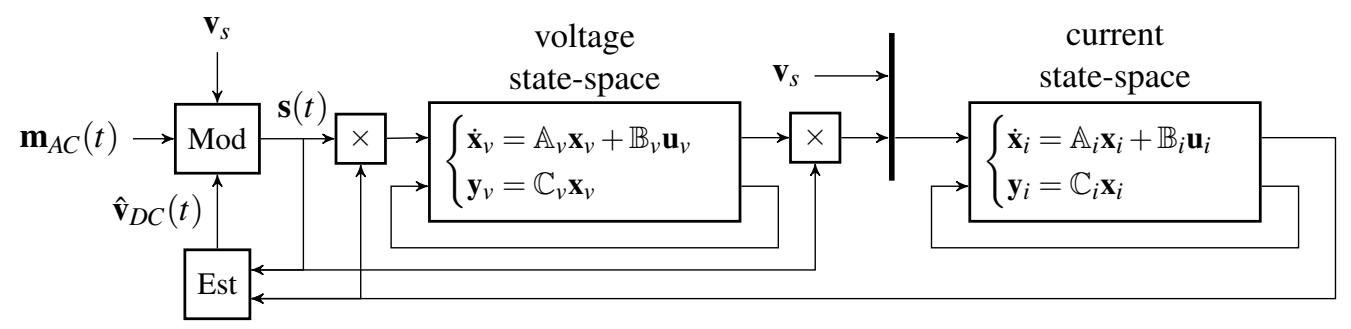

Figure 3: System level state-space representation with cascade of the SM capacitor voltages and state current dynamics, including the estimator for the SM capacitor voltages.
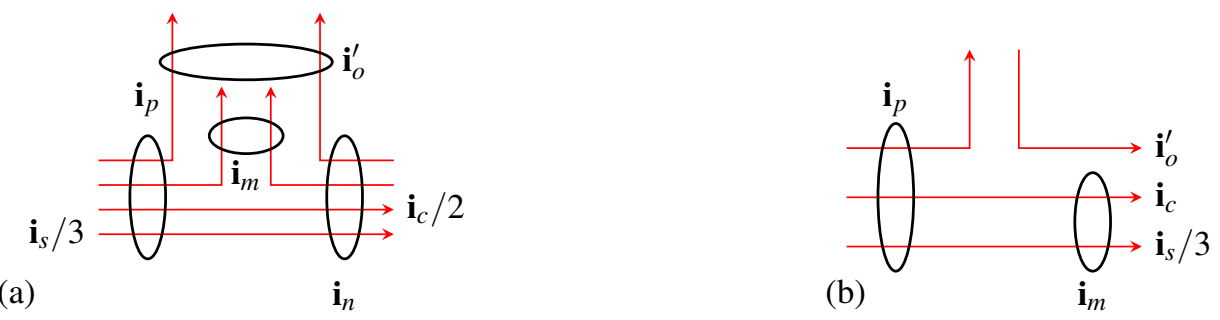

Figure 4: Graphical representation of the current components in both analyzed cases: (a) classical MMC case and (b) OEWMMC case.
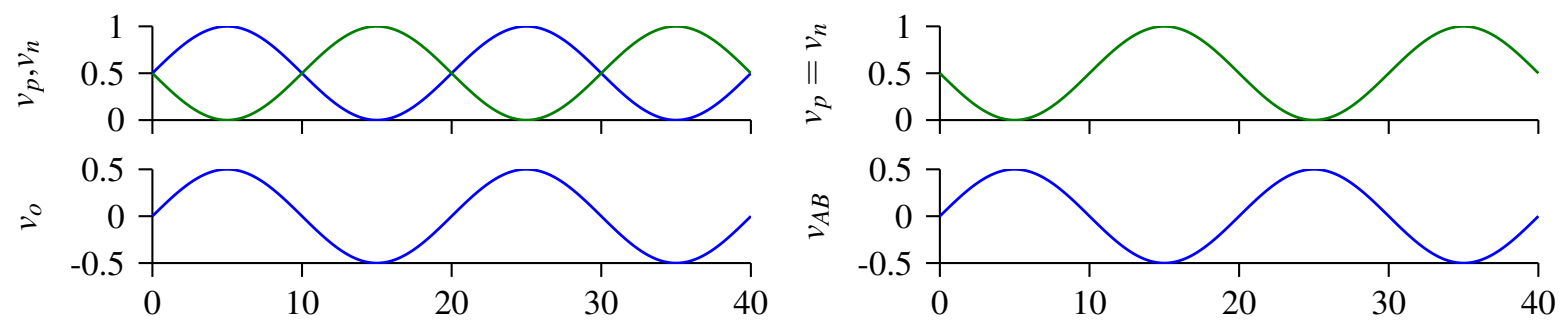

(a)

Time $[\mathrm{ms}]$

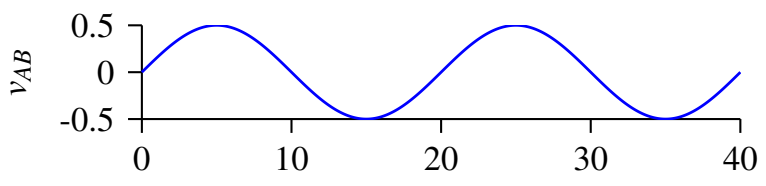

(b)

Time $[\mathrm{ms}]$

Figure 5: Arm reference waveforms in [p.u.] for one MMC phase: (a) classical MMC and resulting mid-point voltage (referenced to bipolar ground) and (b) OEWMMC arms voltages and resulting primary side transformer voltage. 


\section{Simulation results}

Considering that the scope of this paper is not related to the control aspects, simulations are carried out in the continuous-time domain and results are shown in Fig. 6. Due to the infinite switching frequency and identical circuit parameters across all SM, no voltage unbalance appears between the SM capacitor voltages of the same arm. For these reasons only six traces (one per arm) are shown in the top plot of Fig. 6 (a). Similarly, only three traces are visible in the top plot of Fig. 6 (b), as both positive and negative arms of the OEWMMC are modulated in the same way (cf. Fig. 5 (b)). The exact same models could be run with inclusion of switched events at the expense of at least an active balancing control. Differences between SM parameters could be introduced as well, since each SM is modeled by $C_{s m}$ and $R_{s m}$. For the simulations we consider $\mathbf{v}_{g}=0$, as it is a disturbance for the system.

All parameter used for the simulation are indicated in Table II. The SM design is based on available $3.3 \mathrm{kV}$ semiconductors, leading to 6 modules per arm and $1.75 \mathrm{kV}$ across each SM capacitor on average. It is important to stress that the magnetizing inductance has been chosen to achieve a $5 \%$ magnetizing current compared to the nominal output current.

In the side by side comparison shown in Fig. 6, and considering that the modulation signals are different, the dynamics of the SM voltages $\left(\mathbf{v}_{d c}\right)$ changes as well. As already mentioned, only three capacitor voltages are used to describe the OEWMMC, as both positive and negative arms share the same modulation
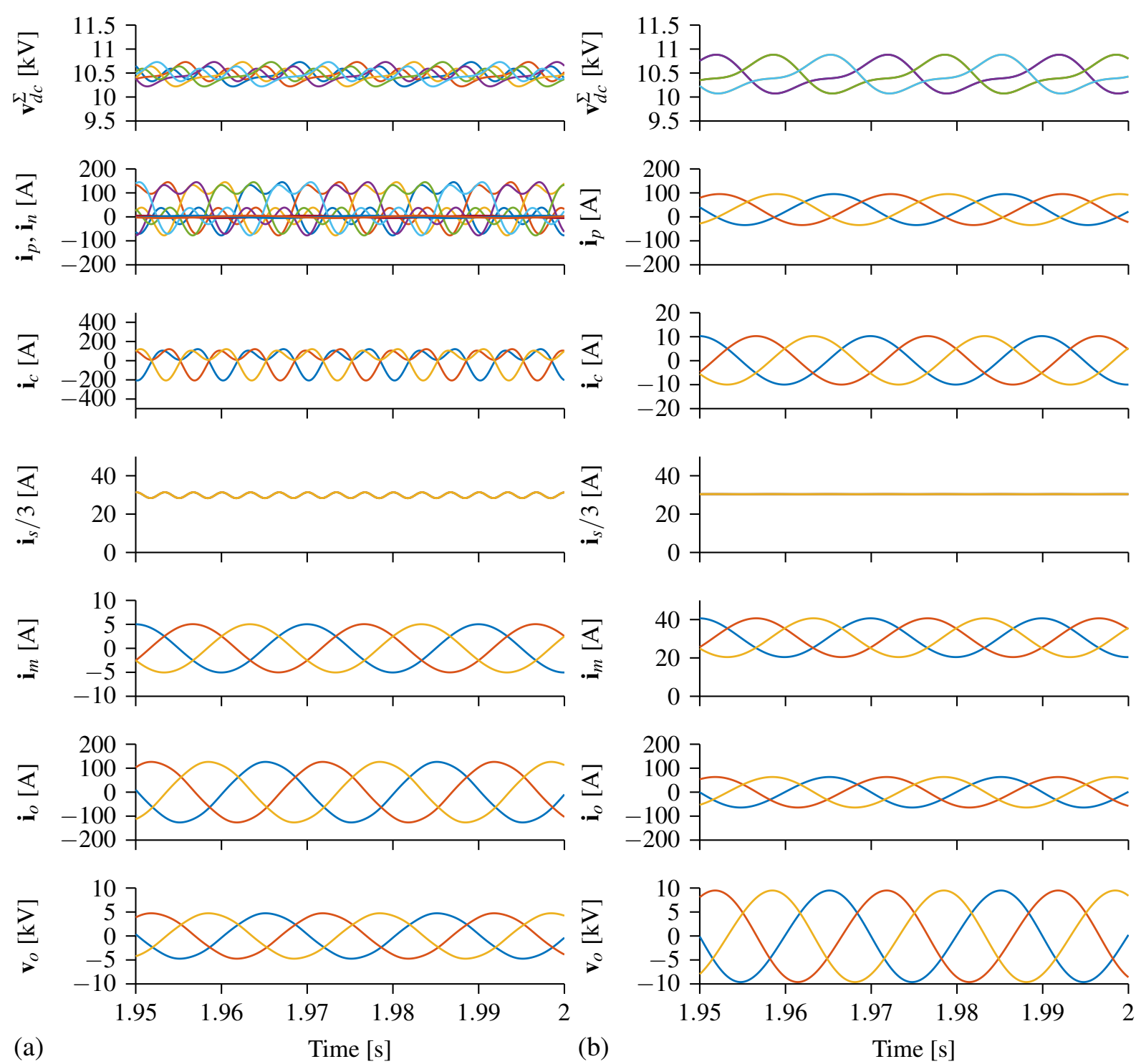

Figure 6: Simulation results: (a) classical MMC with transformer, where $\mathbf{v}_{o}^{T}=\left[v_{A G} v_{B G} v_{C G}\right]$ and (b) OEWMMC, where $\mathbf{v}_{o}^{T}=\left[v_{A B} v_{C D} v_{E F}\right]$. 
Table II: Simulations parameters for a $1 \mathrm{MW}$ converter $\left({ }^{1}\right.$ classical MMC, ${ }^{2}$ OEWMMC).

\begin{tabular}{|c|c|c|c|c|c|c|c|c|}
\hline DC link & \multicolumn{2}{|r|}{ SM } & \multicolumn{2}{|c|}{ Transformer } & \multicolumn{2}{|r|}{$\mathrm{AC}$ grid } & \multicolumn{2}{|c|}{ Classical MMC } \\
\hline $\begin{array}{ll}v_{s} & 10 \mathrm{kV}\end{array}$ & $C_{s m}$ & $1 \mathrm{mF}$ & $N_{1}$ & 1 & $L_{o}$ & $10 \mathrm{mH}$ & $L$ & $100 \mu \mathrm{H}$ \\
\hline $1 \mathrm{mH}$ & $R_{s m}$ & $100 \mathrm{k} \Omega$ & $N_{2}$ & 1 & $R_{O}$ & $37.5 \Omega^{1}, 150 \Omega^{2}$ & $R$ & $1 \mathrm{~m} \Omega$ \\
\hline$R_{s} \quad 2 \mathrm{~m} \Omega$ & $N_{s m}$ & 6 & $L_{m}$ & $3 \mathrm{H}$ & $m$ & 0.95 & & \\
\hline & $V_{d c 0}$ & $1.05 v_{s} / N_{s m} \mathrm{~V}$ & $L_{\sigma}$ & $10 \mu \mathrm{H}$ & & & & \\
\hline & & & $R_{\sigma}$ & $1 \mathrm{~m} \Omega$ & & & & \\
\hline
\end{tabular}

signal (see Fig. 5). Regarding the arm currents properties, both of them have a DC bias equivalent to $\mathbf{i}_{s} / 3$, in accordance to the active power transfer between the DC source and the grid. There is no visible second harmonic component in the OEWMMC case, mainly for loop impedance reasons ( $2 L$ vs. $L_{m}$, cf. Eqs. (12) and (28)). In the classical MMC case, the magnetizing current $\left(\mathbf{i}_{m}\right)$ is without DC bias, while in the OEWMMC case the third of the source DC current is flowing through the magnetizing branch of the transformer. For simulations carried out at $1 \mathrm{MW}$, the input source DC current is around $100 \mathrm{~A}$ or around $30 \mathrm{~A}$ per arm. Finally, as it can be seen from the bottom plot of Fig. 6, the output voltage of the OEWMMC is twice the one of the classical MMC, offering possibilities to match the MMC output voltage with half the DC bus voltage (half the number of cells) at the expense of increased current ratings and reduced voltage resolution. Yet, this comes at a price of non optimal transformer design.

\section{Conclusion}

The paper has presented a formal description of the dynamics of the classical MMC and the OEWMMC with the help of detailed state-space models valid under the condition that all SM voltages are balanced. The parameters relevant for the transformer integration into the MMC structure have been considered throughout the modeling, even though they do not impact the dynamics. Open-loop simulations have been used to highlight the main differences between the classical MMC and the OEWMMC. Despite certain attractiveness associated with the transformer integration in case of OEWMMC, unavoidable presence of large DC bias currents require further analysis of trade-offs at the system level. In particular, transformer design and optimization must be carried out to properly evaluate and quantify these trade-offs.

\section{References}

[1] A. Lesnicar and R. Marquardt. "An innovative modular multilevel converter topology suitable for a wide power range". In: Power Tech Conference Proceedings, 2003 IEEE Bologna. Vol. 3. June 2003, 6 pp. Vol.3.

[2] H.-J. Knaak. "Modular multilevel converters and HVDC/FACTS: A success story". In: Power Electronics and Applications (EPE 2011), Proceedings of the 2011-14th European Conference on. Aug. 2011, pp. 1-6.

[3] M. Glinka and R. Marquardt. "A new AC/AC multilevel converter family". In: Industrial Electronics, IEEE Transactions on 52.3 (June 2005), pp. 662-669.

[4] M. Hagiwara, R. Maeda, and H. Akagi. "Negative-Sequence Reactive-Power Control by a PWM STATCOM Based on a Modular Multilevel Cascade Converter (MMCC-SDBC)". In: Industry Applications, IEEE Transactions on 48.2 (Mar. 2012), pp. 720-729.

[5] A.J. Korn, M. Winkelnkemper, and P. Steimer. "Low output frequency operation of the Modular Multi-Level Converter”. In: Energy Conversion Congress and Exposition (ECCE), 2010 IEEE. Sept. 2010, pp. 39933997.

[6] M. Spichartz, V. Staudt, and A Steimel. "Modular Multilevel Converter for propulsion system of electric ships". In: Electric Ship Technologies Symposium (ESTS), 2013 IEEE. Apr. 2013, pp. 237-242.

[7] G.F. Reed et al. "Ship to Grid: Medium-Voltage DC Concepts in Theory and Practice". In: Power and Energy Magazine, IEEE 10.6 (Nov. 2012), pp. 70-79.

[8] C. Zhao et al. "Power Electronic Traction Transformer - Medium Voltage Prototype". In: Industrial Electronics, IEEE Transactions on 61.7 (July 2014), pp. 3257-3268. 
[9] G.G. Karady, AQ. Huang, and M. Baran. "FREEDM system: An electronic smart distribution grid for the Future". In: Transmission and Distribution Conference and Exposition (T D), 2012 IEEE PES. May 2012, pp. 1-6.

[10] J. Huber and J. W. Kolar. "Volume/Weight/Cost Comparison of a 1MVA 10 kV/400V Solid-State against a Conventional Low-Frequency Distribution Transformer". In: IEEE Energy Conversion Congress and Exposition (ECCE USA 2014), Proceedings of the. Sept. 2014.

[11] M. Hagiwara and H. Akagi. "PWM control and experiment of modular multilevel converters". In: Power Electronics Specialists Conference, 2008. PESC 2008. IEEE. June 2008, pp. 154-161.

[12] A. Antonopoulos, L. Angquist, and H.-P. Nee. "On dynamics and voltage control of the Modular Multilevel Converter”. In: Power Electronics and Applications, 2009. EPE '09. 13th European Conference on. Sept. 2009, pp. 1-10.

[13] S. Ceballos et al. "Analysis of voltage balancing limits in modular multilevel converters". In: IECON 2011 - 37th Annual Conference on IEEE Industrial Electronics Society. Nov. 2011, pp. 4397-4402.

[14] S. Rohner, J. Weber, and S. Bernet. "Continuous model of Modular Multilevel Converter with experimental verification”. In: Energy Conversion Congress and Exposition (ECCE), 2011 IEEE. Sept. 2011, pp. 40214028.

[15] K. Ilves et al. "Steady-State Analysis of Interaction Between Harmonic Components of Arm and Line Quantities of Modular Multilevel Converters". In: Power Electronics, IEEE Transactions on 27.1 (Jan. 2012), pp. 57-68.

[16] J. Reed, G. Venkataramanan, and F. Martinez. "Complex phasor modeling and control of modular multilevel inverters". In: Energy Conversion Congress and Exposition (ECCE), 2011 IEEE. Sept. 2011, pp. 40134020 .

[17] G. Bergna et al. "An Energy-Based Controller for HVDC Modular Multilevel Converter in Decoupled Double Synchronous Reference Frame for Voltage Oscillation Reduction". In: Industrial Electronics, IEEE Transactions on 60.6 (June 2013), pp. 2360-2371.

[18] J. Kolb et al. "Cascaded Control System of the Modular Multilevel Converter for Feeding Variable-Speed Drives”. In: Power Electronics, IEEE Transactions on 30.1 (Jan. 2015), pp. 349-357.

[19] S. Tamada, Y. Nakazawa, and S. Irokawa. "A proposal of Modular Multilevel Converter applying three winding transformer". In: Power Electronics Conference (IPEC-Hiroshima 2014 - ECCE-ASIA), 2014 International. May 2014, pp. 1357-1364.

[20] A. Das, H. Nademi, and L. Norum. "A new circuit topology of Modular Multilevel Converter (MMC) with an open end transformer". In: PCIM Europe 2012; International Exhibition and Conference for Power Electronics, Intelligent Motion, Renewable Energy and Energy Management; Proceedings of. May 2012.

[21] Multilevel converter. WO Patent App. PCT/EP2012/072,757. Jan. 2014.

[22] High voltage dc/dc converter with transformer driven by modular multilevel converters (mmc). WO Patent App. PCT/EP2011/070,629. May 2013.

[23] M.A. Perez and J. Rodriguez. "Generalized modeling and simulation of a modular multilevel converter". In: Industrial Electronics (ISIE), 2011 IEEE International Symposium on. June 2011, pp. 1863-1868.

[24] Q. Tu, Z. Xu, and L. Xu. "Reduced Switching-Frequency Modulation and Circulating Current Suppression for Modular Multilevel Converters". In: Power Delivery, IEEE Transactions on 26.3 (July 2011), pp. 20092017.

[25] A. Rasic et al. "Optimization of the modular multilevel converters performance using the second harmonic of the module current”. In: Power Electronics and Applications, 2009. EPE '09. 13th European Conference on. Sept. 2009, pp. 1-10.

[26] S.P. Engel and R.W. De Doncker. "Control of the Modular Multi-Level Converter for minimized cell capacitance". In: Power Electronics and Applications (EPE 2011), Proceedings of the 2011-14th European Conference on. Aug. 2011, pp. 1-10. 